\title{
Performance Prediction of a Reverse Osmosis Desalination System Using Machine Learning
}

\author{
Divas Karimanzira*, Thomas Rauschenbach \\ Department of Surface Water and Maritime Systems, Fraunhofer IOSB, Ilmenau, Germany \\ Email: *divas.karimanzira@iosb-ast.fraunhofer.de
}

How to cite this paper: Karimanzira, D., \& Rauschenbach, T. (2021). Performance Prediction of a Reverse Osmosis Desalination System Using Machine Learning. Journal of Geoscience and Environment Protection, 9, 46-61.

https://doi.org/10.4236/gep.2021.97004

Received: June 15, 2021

Accepted: July 18, 2021

Published: July 21, 2021

Copyright () 2021 by author(s) and Scientific Research Publishing Inc. This work is licensed under the Creative Commons Attribution International License (CC BY 4.0).

http://creativecommons.org/licenses/by/4.0/

\begin{abstract}
One of the major challenges that membrane manufacturers, commercial enterprises and the scientific community in the field of membrane-based filtration or reverse osmosis (RO) desalination have to deal with is system performance retardation due to membrane fouling. In this respect, the prediction of fouling or system performance in membrane-based systems is the key to determining the mid and long-term plant operating conditions and costs. Despite major research efforts in the field, effective methods for the estimation of fouling in RO desalination plants are still in infancy, for example, most of the existing methods, neither consider the characteristics of the membranes such as the spacer geometry, nor the efficiency and the day to day chemical cleanings. Furthermore, most studies focus on predicting a single fouling indicator, e.g., flux decline. Faced with the limits of mathematical or numerical approach, in this paper, machine learning methods based on Multivariate Temporal Convolutional Neural networks (MTCN), which take into account the membrane characteristics, feed water quality, RO operation data and management practice such as Cleaning In Place (CIP) will be considered to predict membrane fouling using measurable multiple indicators. The temporal convolution model offers one the capability to explore the temporal dependencies among a remarkably long historical period and has potential use for operational diagnostics, early warning and system optimal control. Data collected from a Desalination RO plant will be used to demonstrate the capabilities of the prediction system. The method achieves remarkable predictive accuracy (root mean square error) of $0.023,0.012$ and 0.007 for the relative differential pressure and permeates Total Dissolved solids (TDS) and the feed pressure, respectively.
\end{abstract}

\section{Keywords}

Reverse Osmosis, Membrane Fouling, Fouling Indices, Predicting Models, Machine Learning, Multivariate Temporal Convolutional Neural Networks 


\section{Introduction}

Commercial RO Enterprises, membrane manufacturers and end-users are confronted with two major problems in membrane-based water desalination utilizing reverse osmosis: 1) how to reliably monitor their membrane system performance? and 2) how to detect system performance retardation in real-time due to that membrane fouling and scaling before irreversible membrane processes have happened, which have drastic implications on plant availability, energy, maintenance, and chemical cleaning costs. The current industry-standard performance analysis and evaluation technique are based on trending RO flux decline characteristics of membranes via normalizing system operating data in accordance with the ASTM D-4516 standard method. This method is not sufficient for real-time anomaly detection in system performance. Reverse Osmosis (RO) membrane fouling is a detrimental phenomenon that adversely affects the quantity and quality of the produced water, which are the vital metrics that account for economical and more efficient use of the RO plants. Knowing the status of the system with respect to system performance, membrane fouling or scaling at a very early stage is a prerequisite for positive control and has a direct positive impact on optimizing the total cost and the produced permeate quality (Hoek, Allred, Knoell, \& Jeong, 2008; Vera-Villalobos, Pérez, Contreras, Alcayaga, Avalos, Riquelme, \& SilvaAciares, 2020).

Water managers can utilize the prediction of membrane fouling to make knowledgeable management and financial decisions. However, successful prediction of membrane fouling is very difficult due to many complex factors. For example, membrane characteristics, feedwater composition (nature and concentration of foulants) and operating conditions often have interactions with each other (RuizGarcia \& Ruiz-Saavedra, 2015), which makes the membrane fouling prediction more challenging.

Recently, a great deal of research has been carried out in this field. Several fouling prediction tools and techniques have been developed to describe membrane fouling (Taheri, Sim, Chong, Krantz, \& Fane, 2015; Chai-Hoon, Mohammad, \& Suja, 2015). The traditional and most widely applied fouling indices in RO systems are the Silt Density Index (SDI) and the Modified Fouling Index (MFI). However, these indices lack precision for small foulants (foulant agents < $0.45 \mu \mathrm{m}$ ) (Asif, Tahar, Falath, \& Farooque, 2021; Jin, Lee, Jin, \& Hong, 2017) and do not consider membrane characteristics, feedwater composition (nature and concentration of foulants) and operating conditions. Therefore, recently many studies have focused on the assessment and prediction of membrane fouling using mathematical models, which can provide valuable information on membrane design, operation, maintenance, and process optimization. Unfortunately, it is difficult to isolate and identify the exact cause(s) of fouling in the closed environment of a reverse osmosis system. Mathematically, no single model can successfully predict membrane fouling, which involves various mechanisms and physical/chemical interactions. Especially, the prediction of membrane fouling 
using phenomenological models is obstructed significantly due to the lack of suitable descriptions of the physicochemical phenomena. Modeling techniques based on the direct analysis of experimental data appear to be good alternatives to the techniques used phenomenological hypotheses such as knowledge-based models (Tong, Wu, Wang, Bai, Zhao, Luo, Mao, Ikuno, \& Hu, 2020; Hwang, Oh, Choi, Nam, Lee, \& Choung, 2009). Predictive mathematical models for membrane fouling have not yet proven successful for general use due to the uniqueness of each combination of feedwater composition, membrane type and characteristics, pretreatment methods, and operating conditions. Therefore, several researchers develop models on a case-by-case basis. The transport equations most commonly used for RO are derived using ideal feedwaters of single-component, very soluble salts. Natural waters which serve as feeds to RO systems are inherently very complex chemical systems consisting of many soluble constituents as well as suspended colloidal chemical and biological species (Tang, Chong, \& Fane, 2010). Mathematical models of the feedwater's-chemical composition and water dynamics and membrane characteristics must be coupled with the hydrodynamics of the flow field to accurately describe the fouling system (Karabelas, \& Sioutopoulos, 2015; Shirazi, Lin, \& Chen, 2010). The rates and mechanisms of particle deposition, a variety of possible surface interactions should also be included. Further, capturing long-term dependencies in time series data remains a fundamental challenge (Zhang, Thorburn, Xiang, \& Fitch, 2019; Zhang, Fitch, \& Thorburn, 2020). Machine learning (ML) techniques are increasingly being used to model membrane fouling and RO system performance. Despite advances in building predictive models based on Recurrent Neural Networks (RNNs), these models are still very difficult to scale to very long data sequences, which limits them in identifying the long-range changing patterns, which is critical like in RO system performance variables. Temporal Convolutional Networks (TCNs) overcome these shortcomings by capturing long-range patterns using a hierarchy of temporal convolutional filters (Lea, Flynn, Vidal, Reiter, \& Hager, 2017). Instead of using recurrent structure to maintain temporal dependencies, the TCN applies various sizes of convolutional filters to obtain the temporal dependencies at different time scales. Also, the dilated convolutions (Van den Oord, Dieleman, Zen, Simonyan, Vinyals, Graves, Kalchbrenner, Senior, \& Kavukcuoglu, 2016) increase the receptive field significantly so that long historical data can be utilized.

Furthermore, most RO system researchers build predictive models for each single fouling indicator though all the models indeed deal with the same datasets. Hence, they cannot obtain benefits from each other's learning process. Multi-task learning is applied, which is an essential machine learning paradigm that aims at improving the generalization performance of a task by using other related tasks (Luong, Lee, Sutskever, Vinyals, \& Kaiser, 2016). In the context of RO Membrane fouling prediction, each system variable interacts with and influences other variables in the same ecosystem. The temporal patterns of one system va- 
riable, e.g., transmembrane pressure drop can, therefore, be precious in guiding us in predicting other variables such as permeate flux decline or increase in permeate total dissolved solids (TDS). In this paper, we propose a model based on a multi-task temporal convolution network (MTCN) for predicting multiple RO Membrane fouling indicators, which include the feed pressure, differential pressure, and the permeate TDS. The model can also be used for zero-positive fault diagnostics and system optimization. The key contributions include:

- Besides the feedwater quality and operational data, we also included membrane characteristics and management data in the model.

- A multivariable predictive model to forecast various RO membrane fouling indicators at the same time was developed. Applying a unified model in predicting multiple variables enables the knowledge sharing between multiple learning processes, and also reduces the required computing resources significantly.

- A temporal convolution network (TCN) is applied to learn the long-term temporal dependencies for RO system performance data.

The paper will initially discuss the fouling phenomenon and after an explorative data analysis introduces the methodology for multiple variable predictions.

\section{Methodology}

Figure 1 shows the overall framework of the proposed model. The structure is motivated by the results from (Kanfar, Shaikh, Yousefzadeh, \& Mukerji, 2020). In their experimentation with sequence-based models (i.e., TCN and LSTM), they found out that the models tend to learn the high frequented patterns in a dataset very good compared to the low-frequency patterns. That is why it is reasonable in our case to use the Convolutional Neural networks (CNNs) and the TCN in combination. The authors in (Kanfar, Shaikh, Yousefzadeh, \& Mukerji, 2020) also found out that the TCN learns more stable in comparison to the LSTM. Therefore, the decision was to use the TCN in the framework. It can be seen that our model is divided into four parts. The first part resides the CNNs for feature extraction. Our hope for the Convolutional Neural Network is to capture patterns at different scales by convolving the input with different sized filters. To facilitate training, the dimensions of the multivariate time series are reduced by feeding them into CNNs. The fully connected (FC) layer connecting all the outputs from the CNNs and other inputs serves as the new feature representation of the original multivariate time series. The dataset is split in a second part into subsequences of fixed length by a sliding window which is shaped by the timestep $T$ and the sliding step $S$. The splitted multivariate time series are finally fed into the TCN to train the model. Finally, Bayesian optimization is applied to search for the optimal hyperparameters of TCN. Due to the parallel nature of the problem, multiple searchers with specific search strategy can be applied to efficiently search the entire hyperparameters space in parallel. Applying the framework, the system is trained to make the p-step-ahead predictions. The 


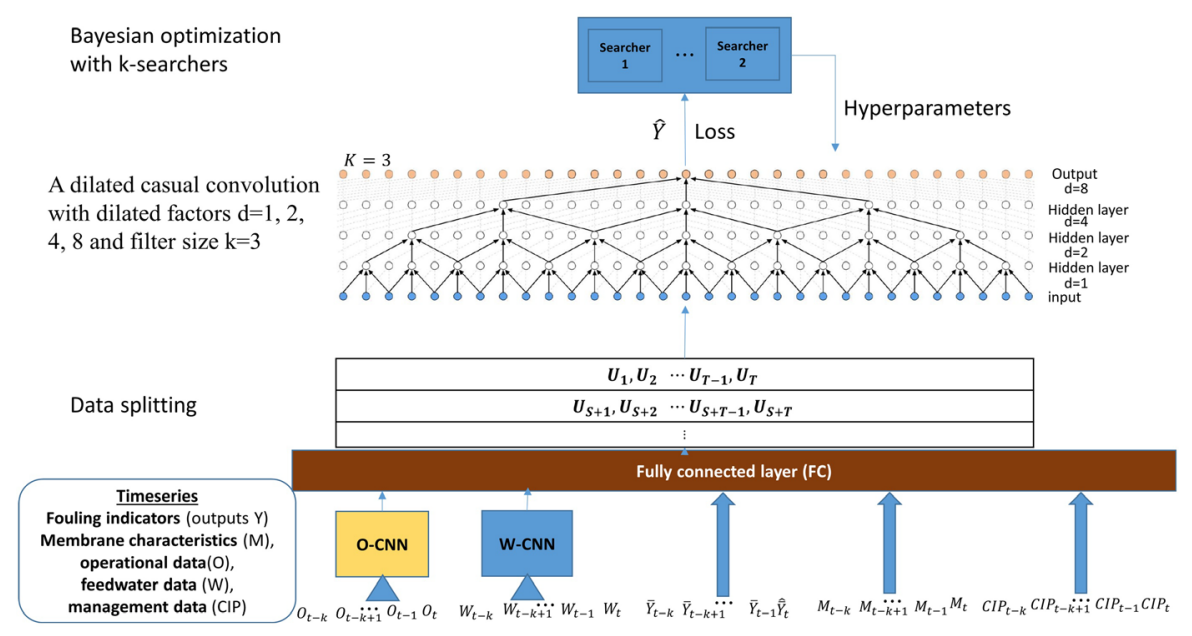

Figure 1. Deep neural network framework for predicting RO system performance.

input layer takes in membrane characteristic data $\left(M \in R^{n \times p}\right)$, feedwater data ( $\left.W \in R^{n \times k_{1}}\right)$, operational data $\left(O \in R^{n \times k_{2}}\right)$, and management data $\left(N \in R^{n \times k_{3}}\right)$ as input. Here, $n$ is the number of observations, $p$ is the number of membrane characteristic variables, $k_{1}$ is the number of feedwater constituents, and $k_{2}$ is the number of operational parameters, and $k_{3}$ is the number of management data components.

The predictive model is based on the multi-task learning paradigm (Baek, Pyo, \& Chun, 2020). The model is designed to learn the temporal dependencies among various RO system variables within a long period of time. The TCN model was adopted due to the two contrasting observations that the relative permeate TDS and relative differential pressure have demonstrated a decreasing trend over time, which could be largely attributed to membrane fouling and management practices. The complete model predicts the relative permeate TDS and relative differential pressure as well as the feed pressure for time $t$ using information from time $t-k$ to $t$. The CNN-TCN models the performance of the RO plant and indirectly membrane fouling in terms of the feed pressure, differential pressure, and permeates TDS. The input parameters of the CNN-TCN model were carefully chosen to include physically meaningful and easy-to-measure membrane operations information. The inputs to the TCN were extracted important features processed by the CNN models for the operating conditions (feed flow rate, feed temperature, and feed pressure) and the feed water quality (feed TDS), the past outputs (the relative permeate TDS and Relative differential pressure data), the management data (operating time, operating time after CIP and the number of CIPs already conducted on the membrane), and a unitless function representing the effect of the size of the feed spacers to elevate the ANN model performance. The use of historical relative permeate TDS and Relative differential pressure data as part of the input allows the TCN model to predict them even without operational or feed water data. Following Box Jenkins optimal one-step prediction, suppose time $t$ is the target time of prediction, then in the test phase, the outputs, relative permeate TDS and relative differential pressure 
at time $t, \hat{Y}_{t}$, can be substituted with $Y_{t-1}$, and the unobserved portion of feedwater data in $W_{t}$ can be substituted with the predicted feedwater data. In the training phase, however, such substitution is unnecessary since all the input data is available. In a TCN model, each predictive task can benefit from the shared hidden representations. The TCN includes a stack of causal convolutional layers. Causal convolution is used to make sure the model will not capture information from the future time index to help the prediction task. In addition, the dilated convolutions and the residual connections are integrated into the TCN to enhance the utilization of long historical observations without the vastly deep structure. By adjusting the dilation factors and filter size, the MTCN can cover a wide range of time series data by applying a hierarchy of filters with various sizes. We Choose larger filter sizes $k$ and increasing the dilation factor $d$, where the effective history of one such layer is $(k-1) \times d$. As is common when using dilated convolutions, we increased exponentially with the depth of the network (i.e., $d=O \times\left(2^{i}\right)$ at level $i$ of the network), which makes sure that some filter that hits each input within the effective history is available, and simultaneously allowing for a large history using deep networks. In addition, the residual connections help to maintain the stability of the deep neural network by enhancing the information flow through the initial layer to the last layer in the deep neural network. The task-specific dense layers with the linear activation function are added on top of the shared convolutional layers.

In the learning, we make use of batch normalization, Adam optimization, and drop out to generalize training predictions to test predictions. The batch size is set to $128.50 \%$ of experimental data are applied for training the CNN-TCN model, which correspond to the first year, The rest of the experimental data are applied for validation to provide an unbiased evaluation of a model fit on the training dataset while tuning model parameters, and to halt training when generalization stops improving. Model testing was performed by using the data in the last year, starting on day $=366$ and forward in time. Thus, model evaluation was carried out in a marching time-series approach with the sliding window.

\section{Evaluation}

In this section, the effectiveness of the proposed method is evaluated by by using the RO system data collected from a large-scale RO plant. The RO plan used for data collection included some pretreatment elements besides the RO processes. To examine fouling, the RO plant was operated automatically in a constant flow mode with a recovery of $35 \%-38 \%$ and data was collected.

\subsection{Data and Exploratory Data Analysis}

Water quality (W) parameters for the feed water to the reverse osmosis (RO) desalination plant were continuously monitored. The data from the RO plant which included five sets (system performance, feed water quality, process data, management data (in this case only CIP), and membrane characteristics data) 
were collected for almost two years, during which Cleaning-in-Place (CIP) was conducted twice. The plant design value of feed TDS and feed temperature was set to $44,000 \mathrm{mg} / \mathrm{L}$ and $15^{\circ} \mathrm{C}$, respectively. All plant operation data and water quality parameters were normalized with plant design parameters. A major part of the Exploratory Data Analysis is searching for relationships between the features and the target. This is important because influencing factors that are correlated to the decision variables are useful to a model for predicting the target variable. Figures 2(a)-(i) show the feed water quality and the operating parameters of the desalination system. Shown in the figure are also the effect $\mathrm{f}$ feed spacer size, Figure 2(a), and the time after cleaning in place (CIP), Figure 2(g).
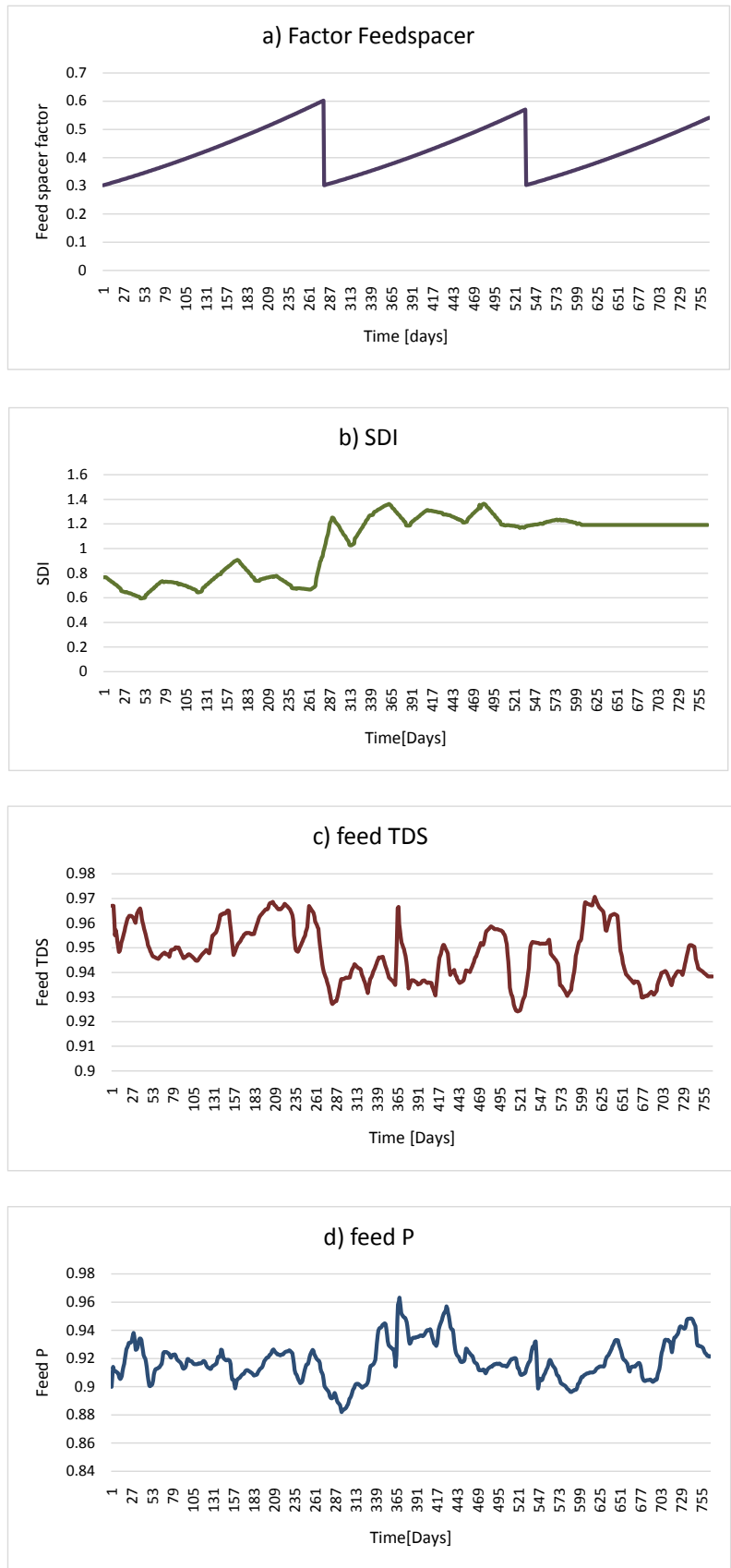

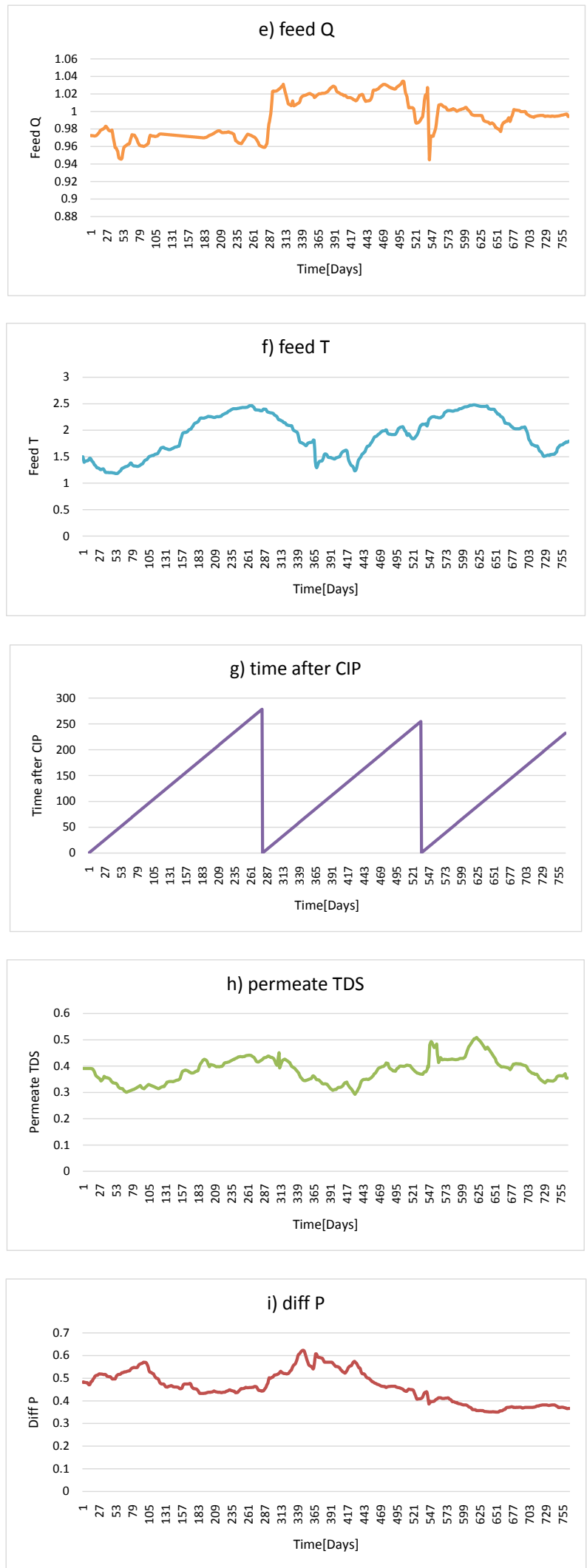

Figure 2. Changes in data with operation time. (a) Feed spacer factor; b) SDI; (c) feed TDS; (d) Relative feed pressure; (e) Relative feed flow rate; (f) relative temperature; (g) time after CIP; (h) Relative permeate TDS; (i) Relative differential pressure. 


\subsubsection{Feedwater Quality}

The feed water quality had two components, feed TDS and feed temperature. As it is shown in Figure 2(c), feedwater TDS remained quite stable (variation = 7.0\%) over the operating time. Otherwise, feed water temperature Figure 2(f) changed drastically in the whole operating period 13 (variation $=140 \%)$. The maximum and minimum relative temperatures were 1.0 and 2.5 , respectively, and the average value was 1.92. The relative feed TDS values ranged from 0.91 to 0.98 . Another important water quality parameter is the Silt density index (SDI). Figure 2(b) shows that after an alteration of the feed water source, the SDI values were considerably increased.

\subsubsection{Operating Data}

The operating conditions of the RO plant normalized with plant design parameters are shown in Figure 2 and had five components, operating time, feed flow rate, feed pressure, differential pressure, and permeate TDS. The changes in feed flow rate, feed pressure, differential pressure, and permeate TDS are shown as functions of the operation time. Since the plant was operated at the constant flux, the permeate flowrate remained stable with time. As shown Figure 2(e), the feed flow rate also did not significantly change (variation $=11.0 \%$, ranging from 0.94 to 0.104 ). Similar to the relative feed flow rate, the relative feed pressure Figure 2 (d) did not change greatly (variation $=13.0 \%$, ranging from 0.87 to 0.98). Contrarily, relative permeate TDS and relative differential pressure changed dramatically as shown in Figure 2(h), and Figure 2(i), respectively.

The relative permeate TDS, Figure 2(h), significantly depended on the feed temperature Figure 2(f). The high temperature increased the salt passage of the RO membrane and thus increased the permeate TDS under constant flux and recovery operating conditions. The relative permeate TDS values ranging from 0.27 to 0.53 . The relative differential pressure, Figure 2(i), also depended on the feed temperature due to viscosity. The low temperature increased the viscosity of feed water and thus increased pressure drop in the pressure vessel. The relative differential pressure values ranged from 0.35 to 0.64 .

\subsubsection{Membrane Properties Data}

Membrane properties were divided into two categories which are the membrane material related and membrane structure-related factors. These factors included hydrophobicity, charge, pore size (thickness of feed spacers), porosity, and pore distribution of membrane. Including the dynamics caused by the Membrane properties such as the feed spacing is one of the novelties of this work. The idea is to use data from literature about effect of membrane feed spacers on the performance with operating time to get a functional relationship.

A study on the Performance of membranes with different thicknesses of feed spacers was conducted in (Park, Cho, Kim, \& Kwon, 2016), where they showed the Normalized Differential Pressure (NDP) of RO filtrations that used 34-mil and 28-mil $(1-\mathrm{mil}=0.0254 \mathrm{~mm})$ feed spacer during a $659 \mathrm{~h}$ operation. In the experiment, the NDP for the two RO vessels was controlled to the same flux and 
recovery as shown from the results in Figure 3(a) for salt rejection of the two options. RO vessel with the 34-mil feed spacer displayed less initial NDP and relatively slow NDP increase up to $264 \mathrm{~h}$ of operation. As shown in Figure 3(b), the specific NDP increase (NDP/h) of the 28 -mil feed spacer case was almost $78 \%$ higher than the 34-mil spacer and took $330 \mathrm{~h}$ to reach 1 bar of NDP compared to $652 \mathrm{~h}$ when 34-mil feed spacers were used. The 34-mil spacers could prolong the filtration time by almost 2 folds. Cleaning-in-place (CIP) was designed to be conducted when the NDP has reached 1 bar. However, the $34 \mathrm{mil}$ spacers caused the NDP to increase slowly so that both the operating conditions and feedwater quality were changed after $264 \mathrm{~h}$ operation to promote RO membrane fouling.

For our CNN-TCN model we utilized the information from the study to develop a unitless function as shown in Figure 4 to take into account the effect of the thickness of feed spacers with respect to time after CIP. This enables the model to be robust to introduction of membranes of other spacer thickness into the desalination plant. As can be seen in Figure 4 a specific polynomial function is created for every known feed spacer thickness. This function saves then as input to the model for a given membrane feed spacer thickness

\subsubsection{Management Data}

The system management data had two components related to cleaning in place, the time after CIP and the number of cleanings conducted already on the membrane. From the data, for 23 months, cleaning in place (CIP) was conducted two
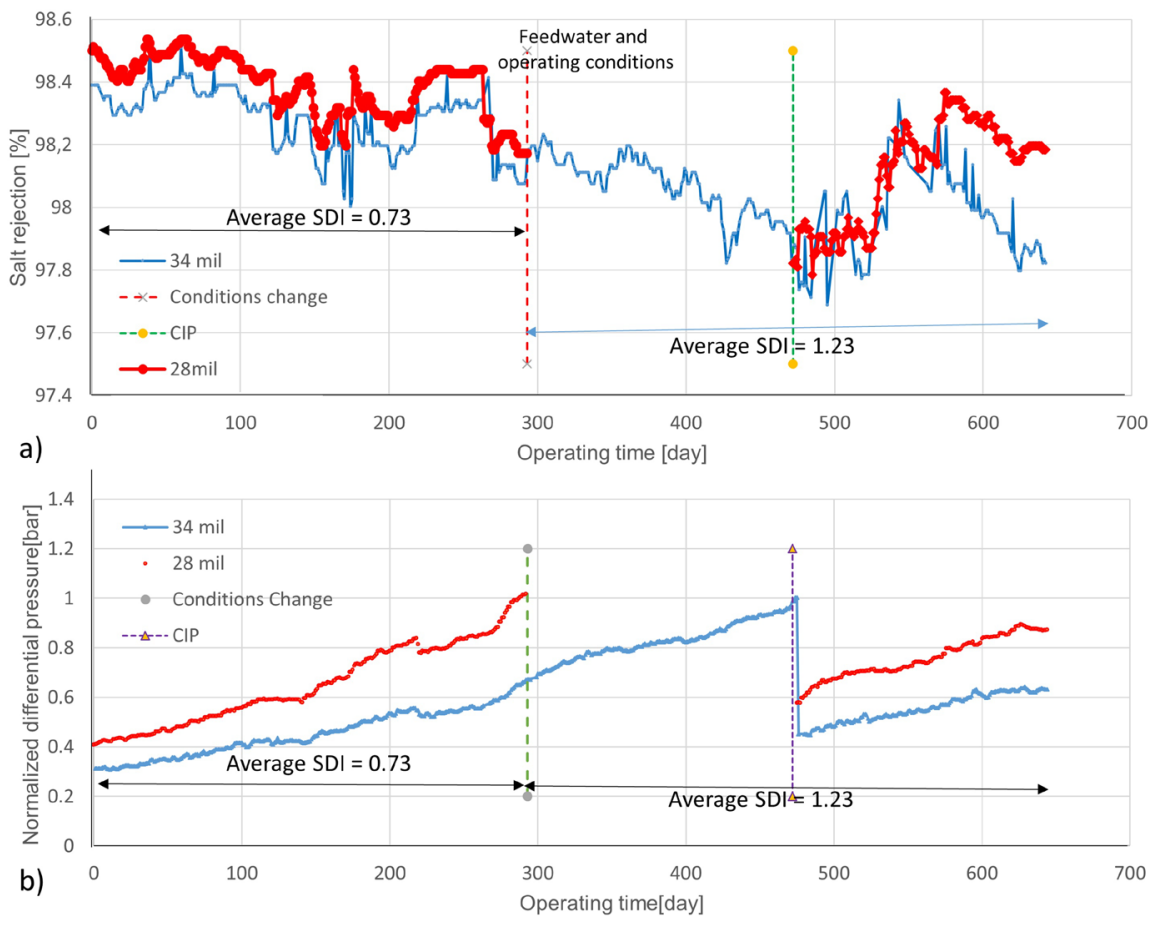

Figure 3. (a) Membrane performance variation (salt rejection) for the two options and (b) NDP for two vessels controlled to the same flux and recovery. 


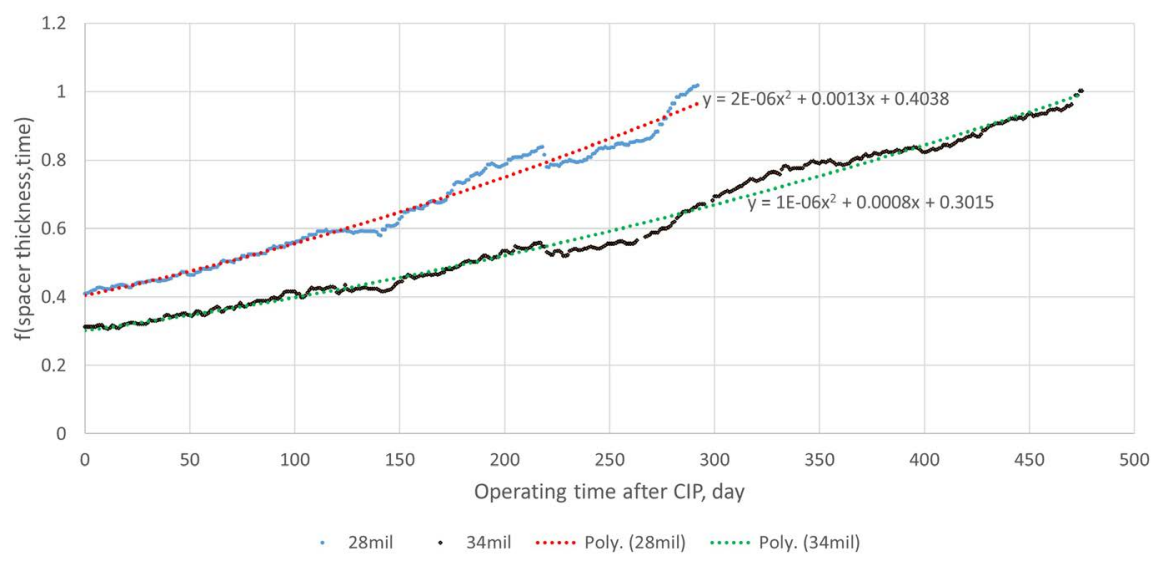

Figure 4. Function of the effect of feed spacer thickness with respect to time after CIP.

times. It can be seen in Figure 4 that the after every cleaning the membrane does not reach its previous maximum performance and degrades with time. To catch this effect in our model we had to use additionally the number of cleanings already conducted on the membrane as an input. An alternative is to use the difference in performance from one cleaning to another, but this requires training data with many cleanings.

\subsubsection{System Performance (Fouling Indicators)}

From this data analysis in Figure 2, it can be seen that the main parameters that indicate a need for CIP (which is in close relationship with fouling) were, the increase in differential pressure between feed side and concentrate side and the permeate TDS. Although relative differential pressure changed dramatically, it did not exceed the CIP criteria. Therefore, the CIP was conducted regularly once every 8 months.

\subsection{Quantitative Determination of the Important Variables}

To quantify relationships between variables, we used the Pearson Correlation Coefficient. This is a measure of the strength and direction of a linear relationship between two variables. A score of +1 is a perfectly linear positive relationship and a score of -1 is a perfectly negative linear relationship. Several values of the correlation coefficient are shown in Figure 5 below. While the correlation coefficient cannot capture non-linear relationships, it is a good way to start figuring out how variables are related. The qualitative relationship stated previously can be clearly seen, for example, the feed temperature is heavily correlated to the permeate TDS with a correlation coefficient of 0.88 and The SDI is strongly correlated to the relative differential pressure and Feed flow rate.

\section{Experimental Setup}

To measure the CNN-TCN model predictive capability, we used the mean absolute error (MAE), the root mean square error (RMSE) as well as the correlation coefficient $\rho$. The model is implemented in Python environment on a PC with 
Intel(R) Core(TM) E5-2620 CPU, 62 GB memory. The training for 50 epochs took $1.45 \mathrm{~s}$ and the prediction for the test data of 365 data points, about $0.045 \mathrm{~s}$. Figure 6 shows the MSE and the number of iterations. A sharp drop in the MSE in the first 9 few iterations is shown. The training cycles stopped after 50, 21, and 25 epochs to preventing over-fitting, with the smallest validation MSE value of $0.00618,0.00012$, and 0.0001 at 50,21 , and 25 iterations, respectively for prediction of relative differential pressure, relative permeate TDS, and relative feed pressure, respectively.

\begin{tabular}{|c|c|c|c|c|c|c|c|c|c|}
\hline i) diff $P$ & 1 & -0.6 & -0.26 & -0.57 & 0.18 & 0.27 & -0.087 & -0.16 & -0.27 \\
\hline h) permeate TDS & -0.6 & 1 & -0.018 & 0.88 & 0.0042 & -0.53 & 0.22 & 0.17 & 0.0071 \\
\hline g) time after CIP & -0.26 & -0.018 & 1 & 0.16 & -0.051 & 0.2 & 0.039 & -0.029 & 1 \\
\hline f) feed $T$ & -0.57 & 0.88 & 0.16 & 1 & 0.035 & -0.53 & 0.12 & 0.19 & 0.18 \\
\hline e) feed $Q$ & 0.18 & 0.0042 & -0.051 & 0.035 & 1 & 0.19 & -0.34 & 0.83 & -0.063 \\
\hline d) feed P & 0.27 & -0.53 & 0.2 & -0.53 & 0.19 & 1 & 0.11 & 0.17 & 0.18 \\
\hline c) feed TDS & -0.087 & 0.22 & 0.039 & 0.12 & -0.34 & 0.11 & 1 & -0.45 & 0.04 \\
\hline b) SDI & -0.16 & 0.17 & -0.029 & 0.19 & 0.83 & 0.17 & -0.45 & 1 & -0.042 \\
\hline \multirow[t]{2}{*}{ a) Factor Feedspacer } & -0.27 & 0.0071 & 1 & 0.18 & -0.063 & 0.18 & 0.04 & -0.042 & 1 \\
\hline & $\begin{array}{l}\stackrel{0}{\frac{0}{4}} \\
\stackrel{\underline{0}}{0} \\
=\end{array}$ & 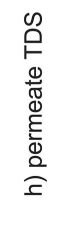 & 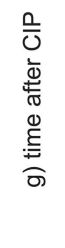 & 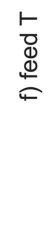 & 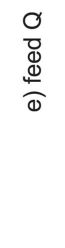 & 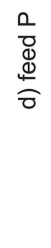 & $\begin{array}{l}\infty \\
0 \\
0 \\
0 \\
\stackrel{0}{0} \\
0\end{array}$ & อิ & 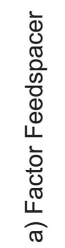 \\
\hline
\end{tabular}

Figure 5. Pearson Correlation Coefficients of the RO plant measured variables.
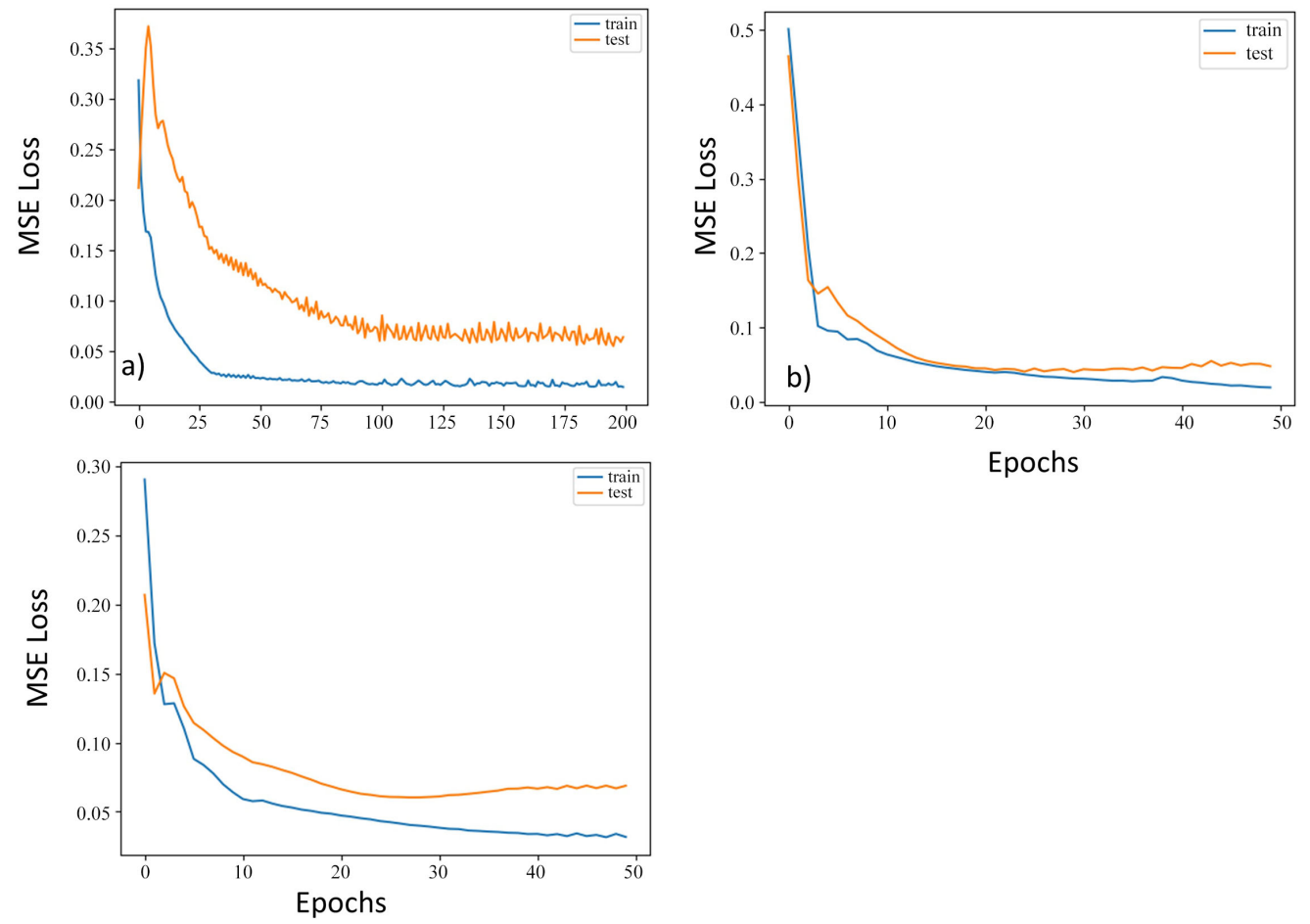

Figure 6. Loss functions of the (a) Relative differential pressure, (b) Relative permeate TDS, and (c) Relative feed pressure. 
The hyperparameters in the prediction model such as the learning rate, batch size, dropout filter size, etc., need to be explored carefully to achieve the best prediction results. We utilize Bayesian optimization to search for these hyperparameters efficiently. From the Bayesian optimization, some of the obtained key hyperparameters are listed in Table 1.

Table 2 illustrates the model performance of the proposed method. Benefiting from the temporal convolutional architecture, dilated convolution, and the residual unit, the method achieves remarkable predictive accuracy for the relative differential pressure and permeates TDS and the feed pressure.

As shown in Figure 7, the method captures the trend of the relative feed pressure; relative differential pressure and relative permeate TDS data in the following 365 days, and also gives the proper estimation when they drop significantly.
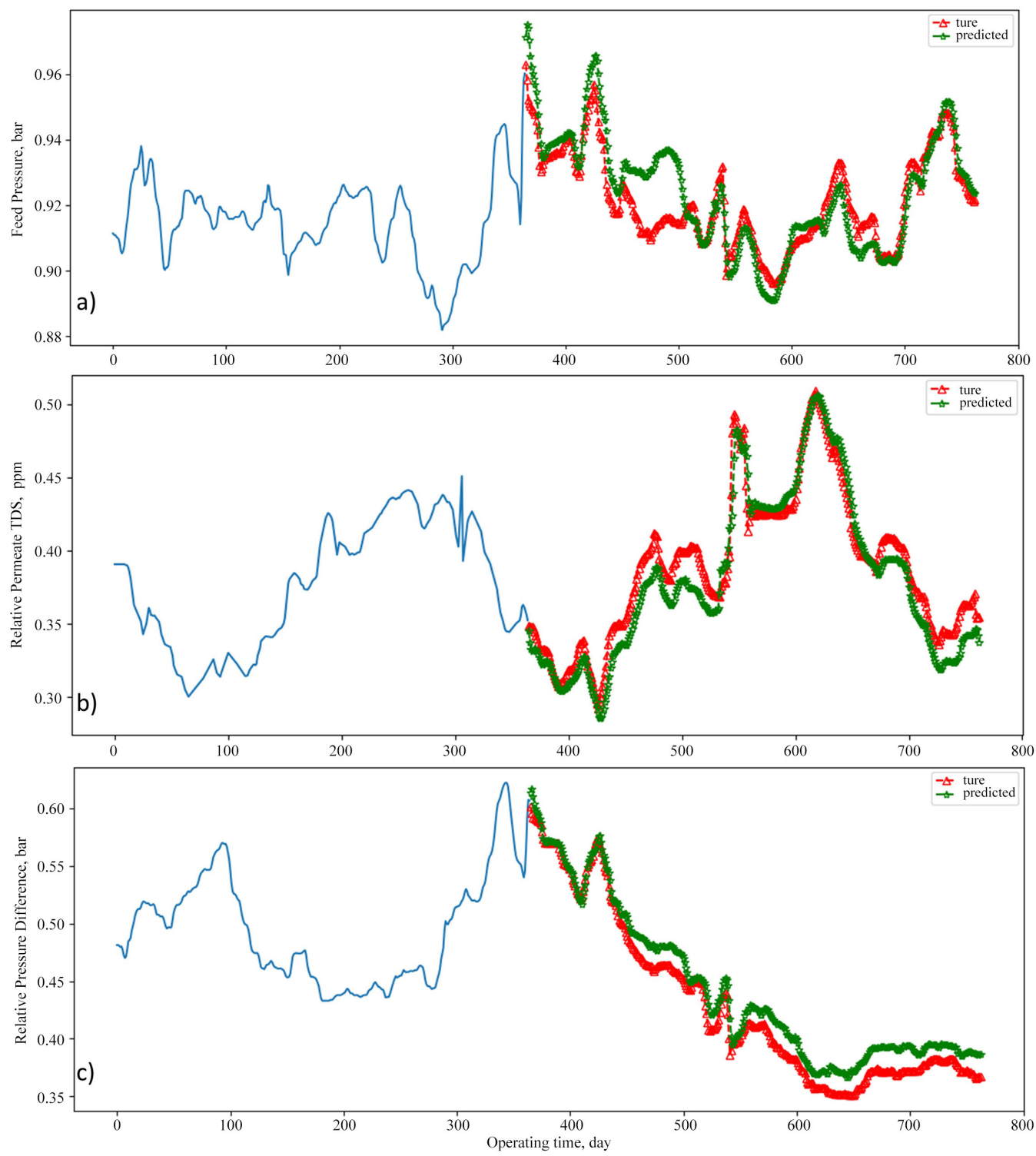

Figure 7. Comparison of experimental data of the full-scale RO plant with the CNN-TCN model results. (a) Relative feed pressure; (b) Relative permeate TDS; and (c) Relative differential pressure. 
Table 1. Key hyperparameters of the CNN-TCN.

\begin{tabular}{cc}
\hline Hyperparameters & Value \\
\hline Number of dense layers & 2 \\
Number of units in dense layers & 64,48 \\
Dilated factors & $1,2,4,8,16,32,64$ \\
Kernel size & 3 \\
Dropout rate & 0.6 \\
\hline
\end{tabular}

Table 2. Model performance on the test data based on two metrics: $\rho$ and rmse.

\begin{tabular}{cccc}
\hline CNN-TCN & Relative differential pressure & RMSE & 0.023 \\
\hline & $\rho$ & 0.996 \\
Relative permeate TDS & RMSE & 0.012 \\
& $\rho$ & 0.983 \\
Relative feed pressure & RMSE & 0.007 \\
& $\rho$ & 0.93 \\
\hline
\end{tabular}

The results from the CNN-TCN model matched the experimental values very well. This suggests that the CNN-TCN model with operating conditions (operating time, operating time after CIP and feed flow rate) and the feed water quality (feed TDS and feed temperature) and the relative permeate TDS and Relative differential pressure data, management data as inputs can successfully fit the operation data from the full-scale RO plant.

\section{Conclusion}

In summary, a methodology based on multi-task temporal convolutional networks for predicting $\mathrm{RO}$ system performance or indirect membrane fouling using multiple variables has been developed. The temporal convolution offers the capability to explore the temporal dependencies among a remarkably long historical period and benefits from each other of the multivariate learning process. Unlike in RNNs where the predictions for later time steps must wait for their predecessors to complete, convolutions can be done in parallel since the same filter is used in each layer. Therefore, long input data sequence can be processed as a whole in a TCN both in training and evaluation, instead of sequentially as in RNN. Experimental results were presented to demonstrate that the proposed method can achieve promising predictive accuracy for long-term RO systems performance prediction. The method can achieve a remarkable predictive accuracy (root mean square error) of $0.023,0.012$ and 0.007 for the relative differential pressure and permeate Total Dissolved solids (TDS) and the feed pressure, respectively. Machine Learning is, after all, data-driven AI, and the model can only as good as the data we have. The method in this paper only uses proxies for fouling as input variables to the model. In the future, it would be interesting to investigate what added value direct measurements, e.g. thickness of cake layer on the membrane, 
number of microbes on the membrane, etc. Ultrasonic methods can be used to measure the thickness and through measurement of carbon dioxide, the number of microbes can be estimated using machine learning methods.

\section{Conflicts of Interest}

The authors declare no conflicts of interest regarding the publication of this paper.

\section{References}

Asif, A., Tahar, L., Falath, W., \& Farooque, M. (2021). Fouling Control in Reverse Osmosis for Water Desalination \& Reuse: Current Practices \& Emerging Environment-Friendly Technologies. Science of the Total Environment, 765, Article ID: 142721. https://doi.org/10.1016/j.scitotenv.2020.142721

Baek, S., Pyo, J., \& Chun, J. A. (2020). Prediction of Water Level and Water Quality Using a CNN-LSTM Combined Deep Learning Approach. Water, 12, 3399. https://doi.org/10.3390/w12123399

Chai-Hoon, C. K., Mohammad, A., \& Suja, F. (2015). Experimental Investigation on Performance of Fouling Prediction Devices for $\mathrm{nf} / \mathrm{ro}$ System. International Journal of Chemical Engineering and Applications, 6, 179-183. https://doi.org/10.7763/IJCEA.2015.V6.477

Hoek, A., Allred, J., Knoell, T., \& Jeong, B.-H. (2008). Modeling the Effects of Fouling on Full-Scale Reverse Osmosis Processes. Journal of Membrane Science, 314, 33-49. https://doi.org/10.1016/j.memsci.2008.01.025

Hwang, T.-M., Oh, H., Choi, Y.-J., Nam, S.-H., Lee, S., \& Choung, Y.-K. (2009). Development of a Statistical and Mathematical Hybrid Model to Predict Membrane Fouling and Performance. Desalination, 247, 210-221. https://doi.org/10.1016/j.desal.2008.12.025

Jin, A., Lee, H., Jin, Y., \& Hong, S. (2017). Application of Multiple Modified Fouling Index (MFI) Measurements at Full-Scale Swro Plant. Desalination, 407, 24-32. https://doi.org/10.1016/j.desal.2016.12.006

Kanfar, R., Shaikh, O., Yousefzadeh, M., \& Mukerji, T. (2020). Real-Time Well Log Prediction from Drilling Data Using Deep Learning. https://doi.org/10.2523/IPTC-19693-MS

Karabelas, A. J., \& Sioutopoulos, D. C. (2015). New Insights into Organic Gel Fouling of Reverse Osmosis Desalination Membranes. Desalination, 368, 114-126. https://doi.org/10.1016/j.desal.2015.01.029

Lea, C., Flynn, M. D., Vidal, R., Reiter, A., \& Hager, G. D. (2017). Temporal Convolutional Networks for Action Segmentation and Detection. IEEE Conference on Computer Vision and Pattern Recognition (CVPR), Honolulu, 21-26 July 2017, 1003-1012. https://doi.org/10.1109/CVPR.2017.113

Luong, M., Lee, Q. V., Sutskever, I., Vinyals, O., \& Kaiser, L. (2016). Multi-Task Sequence to Sequence Learning. CoRR, abs/1511.06114.

Park, H.-G., Cho, S.-G., Kim, K.-J., \& Kwon, Y.-N. (2016). Effect of Feed Spacer Thickness on the Fouling Behavior in Reverse Osmosis Process-A Pilot Scale Study. Desalination, 379, 155-163. https://doi.org/10.1016/j.desal.2015.11.011

Ruiz-Garcia, A, \& Ruiz-Saavedra, E. (2015). 80,000h Operational Experience and Performance Analysis of a Brackish Water Reverse Osmosis Desalination Plant. Assessment 
of Membrane Replacement Cost. Desalination, 375, 81-88. https://doi.org/10.1016/j.desal.2015.07.022

Shirazi, S., Lin, C.-J., \& Chen, D. (2010). Inorganic Fouling of Pressure-Driven Membrane Processes-A Critical Review. Desalination, 250, 236-248. https://doi.org/10.1016/j.desal.2009.02.056

Taheri, A. P. H., Sim, L. N., Chong, T. H., Krantz, W. B., \& Fane, A. G. (2015). Prediction of Reverse Osmosis Fouling Using the Feed Fouling Monitor and Salt Tracer Response Technique. Journal of Membrane Science, 475, 433-444. https://doi.org/10.1016/j.memsci.2014.10.043

Tang, C. T., Chong, H., \& Fane, A. (2010). Colloidal Interactions and Fouling of nf and ro Membranes: A Review. Advances in Colloid and Interface Science, 164, 126-143. https://doi.org/10.1016/j.cis.2010.10.007

Tong, X., Wu, Y. H., Wang, Y., Bai, Y., Zhao, X., Luo, L., Mao, Y., Ikuno, N., \& Hu, H. (2020). Simulating and Predicting the Flux Change of Reverse Osmosis Membranes over Time during Wastewater Reclamation Caused by Organic Fouling. Environment International, 140, Article ID: 105744. https://doi.org/10.1016/j.envint.2020.105744

Van den Oord, A., Dieleman, S., Zen, H., Simonyan, K., Vinyals, O., Graves, A., Kalchbrenner, N., Senior, A., \& Kavukcuoglu, K. (2016). Wavenet: A Generative Model for Raw Audio.

Vera-Villalobos, H., Pérez, V., Contreras, F., Alcayaga, V., Avalos, V., Riquelme, C., \& Silva-Aciares, F. (2020). Characterization and Removal of Biofouling from Reverse Osmosis Membranes (ROMs) from a Desalination Plant in Northern Chile, Using Alteromonas sp. Ni1-LEM Supernatant. Biofouling, 36, 505-515. https://doi.org/10.1080/08927014.2020.1776268

Zhang, Y.-F., Fitch, P., \& Thorburn, P. (2020). Predicting the Trend of Dissolved Oxygen Based on the kPCA-RNN Model. Water, 12, 585. https://doi.org/10.3390/w12020585

Zhang, Y.-F., Thorburn, P., Xiang, W., \& Fitch, P. (2019). SSIM-A Deep Learning Approach for Recovering Missing Time Series Sensor Data. IEEE Internet of Things Journal, 6, 6618-6628. https://doi.org/10.1002/essoar.10500168.1 